\title{
Linx
}

Revue des linguistes de l'université Paris X Nanterre

$45 \mid 2001$

Invariants et variables dans les langues. Études typologiques

\section{Claude Vincenot, Précis de grammaire logique}

Champion, Paris 1998, 1208 pages

\section{Christiane Marque-Pucheu}

\section{(2) OpenEdition}

Journals

Édition électronique

URL : http://journals.openedition.org/linx/845

DOI : $10.4000 /$ linx.845

ISSN : 2118-9692

Éditeur

Presses universitaires de Paris Nanterre

Édition imprimée

Date de publication : 1 décembre 2001

Pagination : 187-188

ISSN : 0246-8743

Référence électronique

Christiane Marque-Pucheu, "Claude Vincenot, Précis de grammaire logique », Linx [En ligne], 45 | 2001, mis en ligne le 25 juin 2012, consulté le 22 septembre 2020. URL : http://journals.openedition.org/linx/ 845 ; DOI : https://doi.org/10.4000/linx.845

Ce document a été généré automatiquement le 22 septembre 2020

Département de Sciences du langage, Université Paris Ouest 


\title{
Claude Vincenot, Précis de grammaire logique
}

Champion, Paris 1998, 1208 pages

\author{
Christiane Marque-Pucheu
}

\section{RÉFÉRENCE}

Claude Vincenot, Précis de grammaire logique, Champion, Paris 1998, 1208 pages.

1 Un lecteur hâtif du Précis de grammaire logique pourrait estimer qu'un pareil intitulé relève du paradoxe. N'est-il pas paradoxal, en effet, d'avoir en mains un ouvrage ne comptant pas moins de 1208 pages et comportant cinq parties organisées en un total de quarante et un chapitres, alors qu'on serait en bon droit d'attendre dudit "Précis », parallèlement à sa précision, son caractère succinct? Le terme «Précis » se justifie en premier lieu par la méthode de description adoptée pour répondre à un ambitieux projet: rendre compte de l'organisation de la langue, voire des langues. Celles-ci seraient régies par un système d'oppositions binaires affectant différents traits, sémantiques, phonologiques, morphologiques et syntaxiques.

2 Un système de notation privilégié est donc mis en œuvre et présenté au début de la Première partie qui tient lieu d'introduction. Ces notations regroupent le carré logique et sa variante défective triangle logique, le cube quand trois propriétés binaires interviennent, le diagramme vectoriel, quand deux propriétés se combinent pour en donner une autre (qui est la résultante), les matrices et les diagrammes de Venn, qui servent théoriquement à représenter des ensembles disjoints, non disjoints ou inclus dans l'autre, et enfin, la représentation sagittale, qui utilise des flèches. Puis ces notations sont utilisées dans les différentes parties de l'ouvrage.

Les deux dimensions de l'axe syntagmatique (Partie II) sont constamment reliées : l'une linéaire, horizontale, avec notamment les fonctions corrélatives anaphorique/ cataphorique, et l'autre hiérarchique, verticale, ou encore "structurale». Un carré logique va servir par exemple à représenter classe et fonction adverbiale par rapport à 
la norme. Au premier sommet du carré logique, skiez intelligemment correspond au modèle canonique où l'ordre (qui est de nature linéaire) et la portée (qui est d'ordre hiérarchique) sont respectés; au second sommet, skiez intelligent établit un écart hiérarchique, un adjectif se trouvant au niveau subverbal et, de ce fait, se voyant assigner une fonction adverbiale; au troisième, dans le célèbre "Je t'aime moi non plus ", l'adverbe non plus crée une distorsion linéaire, puisque habituellement, verbe négatif et non plus sont corrélés, tandis qu'aussi et verbe positif le sont. Et au quatrième sommet, *skiez très établit un écart linéaire en ne précédant pas ici son support (verbal), et un écart hiérarchique en portant sur un verbe, ce qui rompt la distribution complémentaire entre très Adjectif / très Adverbe et beaucoup Verbe.

Exemples d'analyse lexicale (Partie III), la synonymie et l'homonymie sont représentables dans un carré logique à partir des oppositions divergence / convergence et sémique / phonique. Sur les quatre sommets du carré logique, se trouvent respectivement divergence sémique / homonymie divergente, illustrée par le mot grève dans son sens 1 et dans son sens 2, divergence phonique / synonymie divergente illustrée par le couple frêle / fragile), convergence sémique / synonymie convergente illustrée par bécane et bicyclette et, enfin, la convergence phonique / homonymie convergente (mer, mère).

5 Parmi les nombreux points abordés dans l'analyse logico-grammaticale (Partie IV), la fonction nominale sujet vient appuyer l'argument binaire représenté dans un carré logique, avec sujet exprimé plein ou vide (il pleure vs « il pleure dans mon cour comme il pleut sur la ville») et sujet inexprimé plein ou vide (il ne fait que de pleurer vs il ne fait que de pleuvoir).

6 Enfin, dans la Partie V consacrée à la valence verbale, la combinaison des constructions active / passive et de la catégorie grammaticale de perspective prospective / rétrospective s'illustre par un carré logique. La perspective linéaire, qui correspond à une manière d'assumer le discours, est dite prospective quand le discours est assumé dans le sens du déroulement de la chaîne parlée (pousser à) et rétrospective quand elle l'est à rebours (empêcher de). Il en ressort quatre cas de figure: prospectif actif (il l'a poussé à boire), rétrospectif actif (il l'a empêché de boire), prospectif passif (il a été par lui poussé à boire) et rétrospectif passif (il a été empêché de boire par lui).

7 Malgré l'absence d'introduction académique et de cadre théorique annoncé d'emblée, le lecteur aura reconnu sans peine la griffe de Tesnière. Les emprunts terminologiques sont constants, qu'il s'agisse d'actants et circonstants ou d'appellations moins consacrées par ses successeurs, comme antiontif ( $2^{\mathrm{e}}$ personne du verbe) et autoontif ( $1^{\text {re }}$ personne du verbe). À la suite de Tesnière, l'auteur refuse d'établir une hiérarchie entre le sujet et le complément d'objet, rompant en cela avec une tradition qui se perpétue aujourd'hui dans les manuels scolaires. Autre trait partagé, le souci d'articulation entre syntaxe et sémantique à la faveur, notamment, de l'étude des relations hypothétiques. Fidèle à Tesnière, aux antipodes de la théorie distributionnelle qui a pour objectif de procéder de manière inductive à partir d'un corpus, Claude Vincenot travaille sur des exemples fabriqués. Sur le mode de représentation adopté pour les phénomènes linguistiques, Claude Vincenot se démarque de Tesnière. Il se démarque aussi des descriptions utilisables informatiquement : sans négliger totalement les matrices qu'il propose parallèlement aux carrés logiques, il semble donner à ces derniers la prédilection, leur caractère plus imagé étant indéniable. Enfin, la notion de perspective, 
hissée au rang de catégorie grammaticale, plus guillaumienne, est reprise notamment à Adamczewski.

8 Tout au long de l'ouvrage, de nombreux indices attestent d'un vif intérêt pour les mathématiques : le signe $x$, les termes de "projection ", "relèvement » et le doublon fréquent «définition/propriétés». Les notions de rapport entre deux unités linguistiques sont relativement intuitives et employées à bon escient. Les cas où il est proposé d'établir un rapport entre deux relations binaires étant nombreux, une représentation sous forme de carré fournit une représentation graphique pratique. Les formes défectives où l'une des combinaisons est interdite ou bien inexistante sont alors représentées sous la forme d'un triangle. Mais d'autres schématisations ne sont pas aussi claires, ainsi la notation de rapport des propriétés d'une multiplication, qui n'est absolument pas définie. Il ne faut pas se méprendre non plus sur le sens du titre «logique »: il n'est nullement question de mathématique et de logique au sens traditionnel et, souvent, cet ouvrage a de mathématiques et de logique surtout dans la terminologie. Certaines notions utilisées relèvent plutôt du bon sens que des mathématiques, telle celle d'ensemble. Le bien-fondé de certaines représentations peut être soulevé, tel ce carré logique (p. 247) qui devrait sans doute être un triangle. Les diagrammes de Venn ne paraissent pas toujours utilisés pertinemment, comme celui de la page 584 qui n'est ni explicatif ni exact dans sa représentation. En effet, il présente une vue symétrique des deux concepts (oiseau/avion) à la première et à la deuxième étape, mais aux étapes 3 et 4 la symétrie est rompue : pourquoi un avion peut être aussi un coucou et pourquoi un oiseau ne serait pas, par exemple, aussi un aéronef ? Enfin, les "faits" sont souvent supposés connus et non argumentés : ainsi, dans Cela nous déplaît qu'on nous reprenne, cela est dit cataphorique et corrélé au sujet qu'il représente et dans Il ne nous plaît pas qu'on nous reprenne, il est pronom sujet vide, « syntaxiquement corrélé au sujet qu'il annonce sans le représenter ", ce qu'aurait démontré l'opposition On nous reprend. Cela nous déplaît / On nous reprend. *Il ne nous plaît pas. A cela s'ajoutent des erreurs orthographiques comme tu te rassure (p. 86), auxilliaire (p. 239), catophorique (p. 331), factultatif (p. 260), le choix et possible (p. 756), un astérisque sans objet devant $I l$ va à Paris (p. 263), la syllabe ai donnée pour ain (tableau p. 530), ou encore une virgule dans le membre de phrase (donné hors contexte) The car, I have seen (p. 184), qui serait censée indiquer une pause ( ?).

9 Mais n'est-il pas injuste de comptabiliser des erreurs dans un ouvrage qui a l'insigne mérite de donner un tableau descriptif complet de la langue, une fois que le lecteur s'est familiarisé avec les nombreux termes techniques? Cela suffit pour recommander sa lecture. 